Gut, 1973, 14, 835-841

\title{
The Lundh test in the diagnosis of pancreatic disease : A review of five years' experience
}

\author{
A. MOTTALEB ${ }^{1}$, F. KAPP ${ }^{2}$, E. C. A. NOGUERA ${ }^{3}$, T. D. KELLOCK, \\ H. S. WIGGINS, AND SHEILA L. WALLER \\ From the Medical Research Council Gastroenterology Unit and Department of Gastroenterology, \\ Central Middlesex Hospital, London
}

SUMMARY An analysis of 523 Lundh tests performed on 492 patients over a five-year period has been made. The results suggest that the test is a simple, cheap, and reliable procedure for the diagnosis of pancreatic exocrine insufficiency, and is suitable for both specialized units and general hospitals. It is particularly useful in the differential diagnosis of patients with steatorrhoea or obstructive jaundice and can often distinguish between malignant and benign lesions, as well as indicating the site of the obstruction. It is of lesser value in the diagnosis of abdominal pain.

The measurement of the mean tryptic activity in duodenal juice following a standard liquid test meal (Lundh, 1962) has been shown to be a useful index of exocrine pancreatic function in selected patients (Lundh, 1962; Cook, Lennard-Jones, Sherif, and Wiggins, 1967; Levin, Youngs, and Bouchier, 1972; Lurie, Brom, Bank, Novis, and Marks, 1973). The encouraging results obtained in this department (Cook et al, 1967) led us to adopt the Lundh test for the assessment of pancreatic function. We now review our experience of 523 tests carried out in the course of routine diagnostic investigation on unselected patients with a variety of gastrointestinal symptoms over a five-year period. The object of this survey was to assess the value of the routine use of the Lundh test in the light of the earlier encouraging reports.

\section{Methods}

Patients were fasted overnight and received no drugs on the day of the test. Details of the Lundh test and of estimation of the mean tryptic activity using the synthetic substrate alpha-N-benzoyl-L-arginine-ethyl ester (BAEE) have been described (Cook et al, 1967; Wiggins, 1967) and were followed with two minor

\footnotetext{
${ }^{1}$ A.M. was in receipt of a Nuffield fellowship

F.K. was in receipt of a Frank Lichtenstein bursary from the University of Basel

'E.C.A.N. was in receipt of a fellowship from the Fundacion Para El Progreso de la Medicina (Hospital Privado, Córdoba, Argentina) and a grant from the Central Middlesex Hospital Research Fund.

Address for reprints: S.L.W., Medical Research Council Gastroenterology Unit, Central Middlesex Hospital, London, NW10 7NS.

Received for publication 17 July 1973.
}

modifications: flavouring of the test meal was omitted so that the colour of the aspirate could be recorded, and the mean tryptic activity of the complete two-hour collection only was measured. The volume and appearance of the juice and the position of the tube were carefully noted and recorded on a special chart at the time of the test.

The notes of all patients who had a Lundh test between 1 January 1967 and 31 December 1971 have been reviewed and the clinical, biochemical, radiological, and Lundh test data were abstracted on to sorting cards. There was no selection of cases, the clinicians using the test in the routine investigation of suspected pancreatic disease. Patients in whom the Lundh test was not satisfactory or whose notes could not be traced were excluded, and the remainder were grouped according to the clinical diagnosis obtaining at the time the notes were reviewed.

An absolute diagnosis of pancreatic disease may be difficult either to establish or to refute. For the purposes of the present assessment, therefore, the following criteria were adopted and were completely independent of the result of the Lundh test. Nonpancreatic patients were those in whom ultimately no evidence of pancreatic disease was found. The non-pancreatic patients were further subdivided into three groups: controls, diabetics without steatorrhoea or pancreatic calcification, and patients who had undergone gastric surgery. It is known that diabetics may have diminished pancreatic exocrine function (Chey, Shay, and Schulman, 1963), whilst gastric surgery may affect pancreatic function (Wormsley, 1972a, b).

Pancreatic disease was considered to be 'proven' 
if there was either (1) $x$-ray evidence of pancreatic calcification, (2) operative or necropsy findings of pancreatic disease, or (3) if the serum amylase was higher than $1100 \mathrm{IU} / 1$ during an attack of pain. For the purpose of this study patients who fulfilled criteria 1 and 2 and did not have carcinoma of the pancreas were designated as 'chronic pancreatitis'. Patients in group 3 were classified as having acute or relapsing pancreatitis, depending on whether they had one, or more than one, attack of pain associated with an elevated serum amylase. Patients were assigned to the 'suspected' pancreatic disease group if they had clinical features or barium meal studies suggestive of pancreatic disease, but had none of the criteria of 'proven' pancreatic disease as described above and no definitive diagnosis had been made.

The data were analysed first within these clinical categories, each patient being represented in the figures by one value only; the most recent result was used for those patients who had more than one test. However, because the presenting symptom is important in making a diagnosis, a further analysis with regard to the presenting symptom was performed (Cook et al, 1967), each patient being considered with respect to only one symptom. Where more than one symptom was present jaundice took precedence over steatorrhoea and steatorrhoea over pain. The results of serial Lundh tests in the same patient were analysed with respect to time and clinical features.

\section{Results}

Five hundred and twenty-three tests were performed during the five-year period on 492 patients. Fiftyeight patients were excluded from the analysis either because the notes could not be traced ( 26 patients), or because the test was technically unsatisfactory (32 patients, table I). Of the 434 patients included in this study, 27 had 31 further tests of which six were unsatisfactory, making the technical failure rate $7 \%$ (38/523 tests, table I).

\section{ANALYSIS WITH REGARD TO THE CLINICAL DIAGNOSIS}

\section{Non-pancreatic conditions}

Two hundred and thirty-five patients had no evidence of exocrine pancreatic disease, were not diabetic, and had not had gastric surgery: these form the control group (table II). As there was no difference in the distribution of the mean tryptic activity in the various diagnostic categories within this control group (fig 1), the results were pooled. The combined data for the control group had a log-normal distribution, with a mean of $16.8 \mathrm{IU} / 1$ and a range of

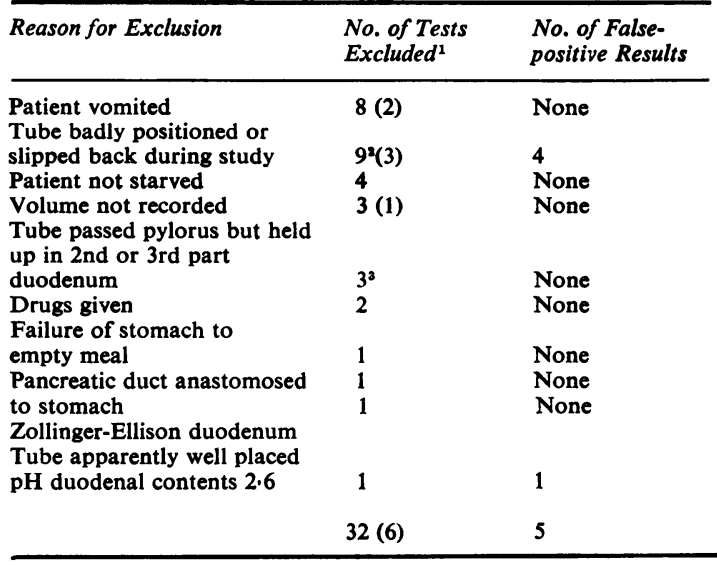

Table I Results of Lundh tests

${ }^{1}$ Nos. in brackets = patients in whom a repeat test was available during five-year period

${ }^{2}$ Two of these patients had a repeat test after the end of the five-year period.

${ }^{3}$ All these patients had carcinoma pancreas.

\begin{tabular}{|c|c|c|c|c|}
\hline Excluded & $\begin{array}{l}\text { Notes not traced } \\
\text { Test unsatisfactory }\end{array}$ & $\left.\begin{array}{l}26 \\
32\end{array}\right\}$ & 58 & 58 \\
\hline $\begin{array}{l}\text { Non-pancreatic } \\
\text { disease }\end{array}$ & $\begin{array}{l}\text { Controls } \\
\text { After gastric surgery } \\
\text { Diabetes }\end{array}$ & $\left.\begin{array}{r}235 \\
40 \\
29\end{array}\right\}$ & \multicolumn{2}{|l|}{304} \\
\hline $\begin{array}{l}\text { Carcinoma } \\
\text { biliary tree }\end{array}$ & $\begin{array}{l}\text { Bile Ducts } \\
\text { Hepatic Ducts } \\
\text { Gallbladder }\end{array}$ & & \multicolumn{2}{|l|}{8} \\
\hline $\begin{array}{l}\text { 'Proven' } \\
\text { pancreatic } \\
\text { disease }\end{array}$ & $\begin{array}{l}\text { Acute pancreatitis } \\
\text { Chronic pancreatitis } \\
\text { Relapsing pancreatitis } \\
\text { Carcinoma head of } \\
\text { Pancreas }\end{array}$ & $\begin{array}{r}4 \\
29 \\
16 \\
23\end{array}$ & \multirow[t]{2}{*}{77} & \multirow[t]{2}{*}{434} \\
\hline $\begin{array}{l}\text { 'Suspected' pan- } \\
\text { creatic disease }\end{array}$ & $\begin{array}{l}\text { Carcinoma body of } \\
\text { pancreas }\end{array}$ & 5 & & \\
\hline \multicolumn{2}{|c|}{ Total number of patients } & & & 492 \\
\hline
\end{tabular}

Table II Patients grouped according to clinical diagnosis

7.0 to $38.0 \mathrm{IU} / 1$ (calculated from the mean $\pm 2 \mathrm{SD}$ ). Mean tryptic activity of $6 \mathrm{IU} / 1$ or less was therefore indicative of pancreatic exocrine insufficiency.

A further 69 patients (fig 2) with no clinical or other evidence of pancreatic exocrine disease had either diabetes (29) or had undergone gastric surgery (40). As was anticipated, a few of these (two diabetics and six after gastric surgery) had abnormal mean tryptic activity.

Pancreatic disease and biliary tract carcinoma Of the 77 patients with 'proven' pancreatic disease (including acute and relapsing acute pancreatitis), $46(60 \%)$ had an abnormal mean tryptic activity; 


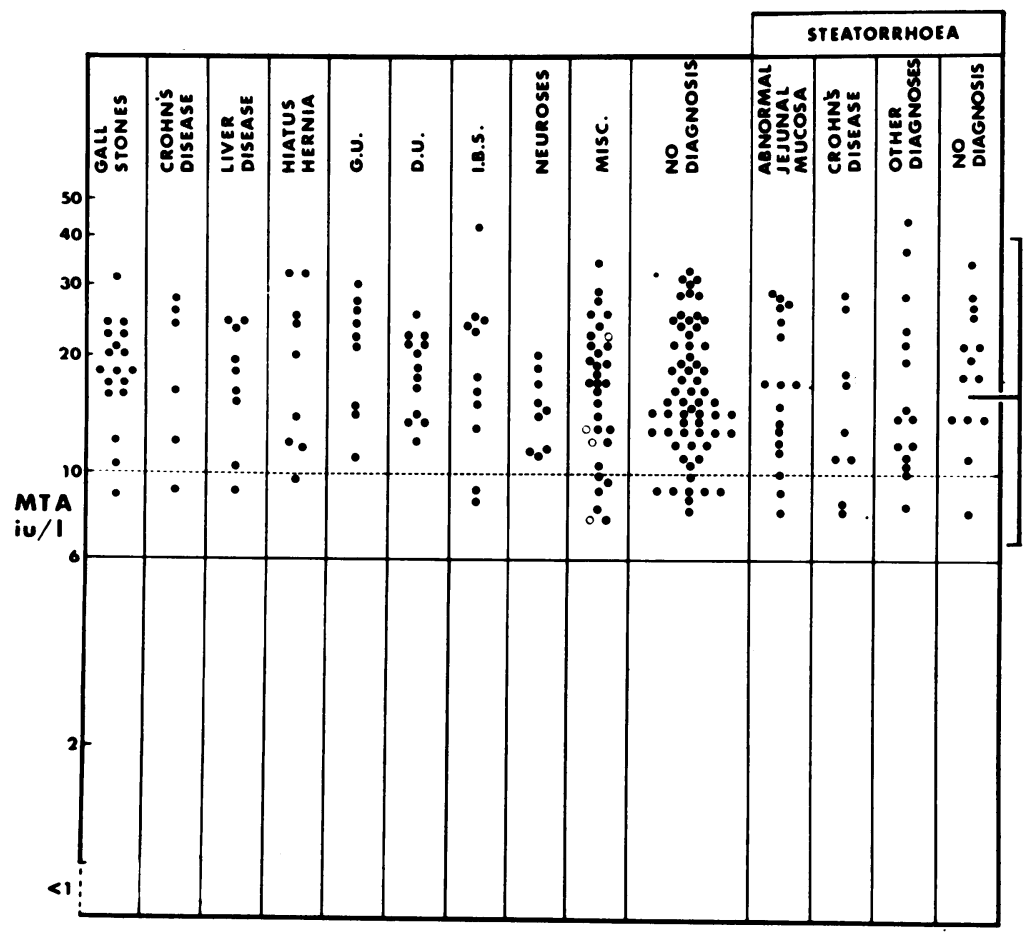

Fig 1 Mean tryptic activity (MTA) (plotted on a log scale inall figures) for the 235 control subjects according to diagnosis. Horizontal bars to the right of graph represent the mean \pm $2 S D$ for the whole group. $\mathrm{O}=$ patients with anabnormal jejunal mucosa but no recorded steatorrhoea.

Dotted line represents published lower limit of normal for healthy controls (Cook et al, 1967). The thick line is the lower limit of normal for patients in the present study. These lines have the same meaning in all following figures.

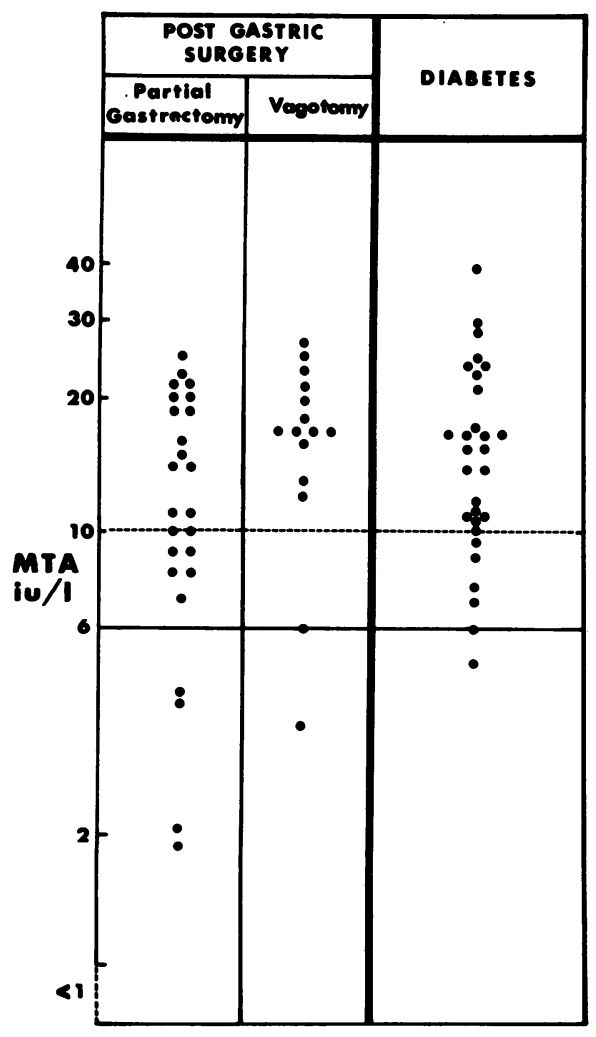

Fig 2 Mean tryptic activity in 40 patients after gastric surgery and 29 diabetics.

if, however, only those patients with chronic pancreatitis or carcinoma of the pancreas were considered, the proportion with a mean tryptic activity of six or less rose to $71 \%$ (fig 3). Seventeen of the 23 patients with carcinoma of the head and three of five patients with carcinoma of the body of the pancreas had an abnormally low mean tryptic activity, whereas all the eight patients with biliary tract carcinomas had a normal mean tryptic activity. Nineteen of the 29 patients with chronic pancreatitis and 6 of the 16 patients with relapsing pancreatitis also had an abnormal mean tryptic activity. In the 'suspected' pancreatic disease group there were 10 patients who, on clinical grounds, clearly had pancreatic disease and all but one of these patients had an abnormal mean tryptic activity (fig 3 ).

\section{ANALYSIS WITH REGARD TO THE PRESENTING SYMPTOMS}

\section{Jaundice}

The mean tryptic activity of the 61 patients presenting with jaundice are in figure 4. All eight patients with biliary tract carcinoma and 14 of 23 patients with carcinoma of the head of the pancreas presented with jaundice. Seventy-one per cent of those with pancreatic carcinoma, but none of those with carcinoma of the biliary tract, had an abnormal mean tryptic activity. Four of the 16 patients with obstructive 


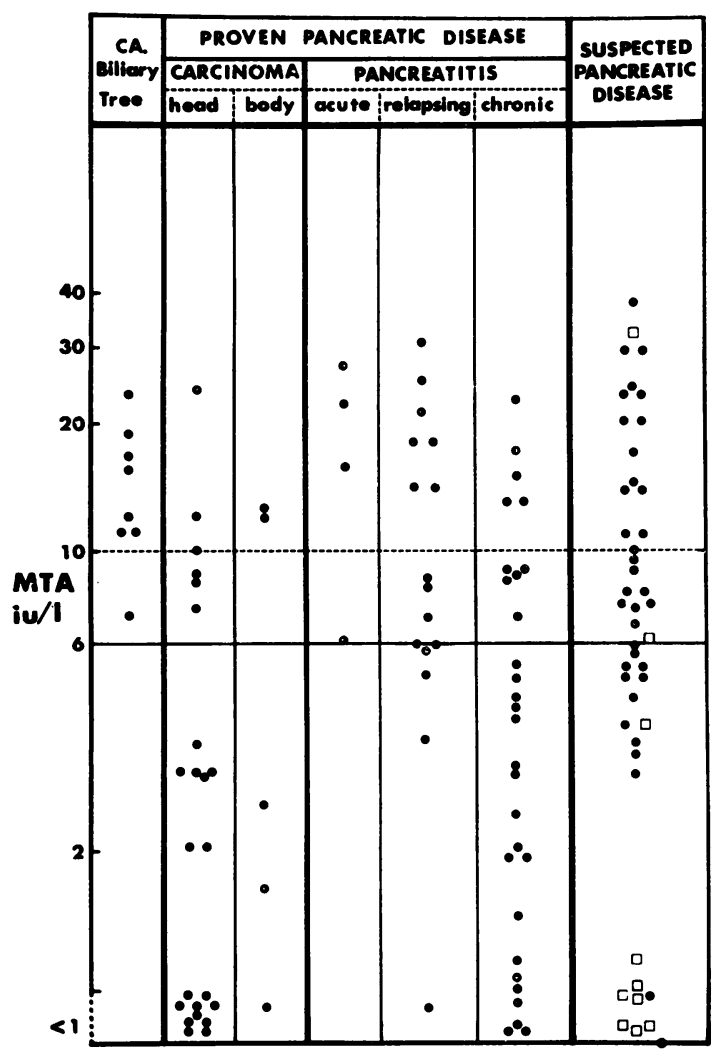

Fig 3 Mean tryptic activity in patients with carcinoma of biliary tree (8); 'proven' pancreatic disease (77); and 'suspected' pancreatic disease (45) including $\square=$ patients in whom a firm diagnosis was made on clinical grounds but who did not fulfil the criteria for 'proven' pancreatic disease.

jaundice due to gallstones had an abnormally low mean tryptic activity and one a borderline value but all five patients also had 'proven' chronic pancreatitis. The only other abnormally low mean tryptic activity in this group was in a patient with liver metastases following resection for gastric carcinoma. His jaundice was due to obstruction of the bile duct by a secondary carcinoma in the duodenum close to the ampulla of Vater.

The appearance of the duodenal contents proved to be of great diagnostic value in this group of patients. Sixty-five per cent of patients with jaundice caused by malignant lesions had no bile in the duodenal contents at any time during the test compared with only $5 \%$ of patients with nonmalignant lesions. Frank blood was present in four of the 14 cases of carcinoma of the head of the pancreas.

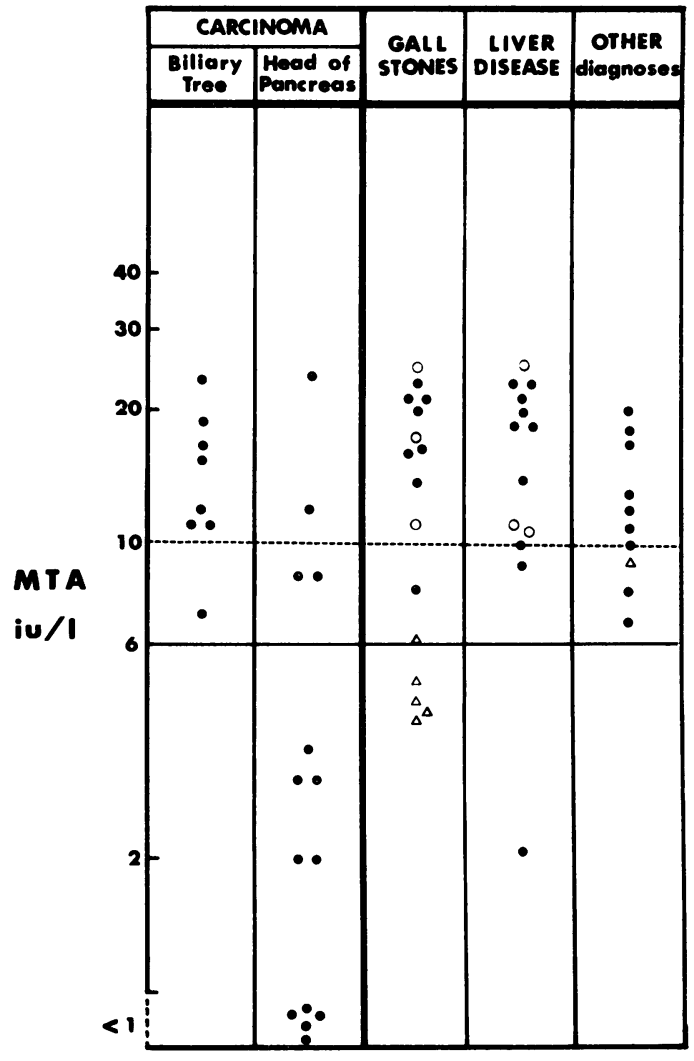

Fig 4 Mean tryptic activity in all patients presenting with jaundice. $O=$ diabetes $\Delta=$ 'proven' chronic pancreatitis. Other diagnoses include five patients with 'suspected' pancreatic disease.

\section{Steatorhoea}

There was virtually no overlap between patients with proven pancreatic disease in whom other major causes of steatorrhoea had been excluded and those in whom steatorrhoea was not due to pancreatic disease (fig 5). All but one patient with 'proven' pancreatic disease had an abnormal Lundh test. The mean trypsin activity values were well below the lower limit of normal (6IU/1) and were greater than $2 \mathrm{IU} / 1$ in only five of the 16 patients in this group. It is interesting that of these five, two had other possible causes for steatorrhoea (gastric surgery and a pancreatoduodenectomy for Crohn's disease) and in the remaining three other causes had not been excluded. It is noteworthy that most patients with clinically 'suspected' but not 'proven' pancreatitis and steatorrhoea also had very low levels of mean tryptic activity (fig 5). By contrast, all patients with steator- 


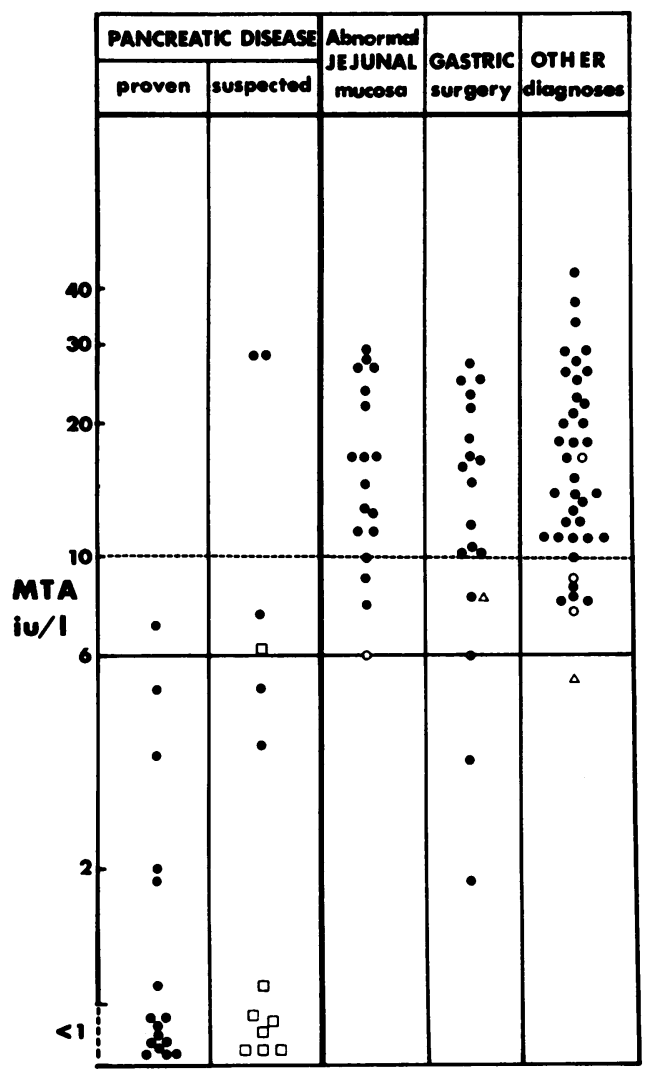

Fig 5 Mean tryptic activity in all patients presenting with steatorrhoea. $\Delta=$ patients with 'proven' pancreatic disease but another possible cause for steatorrhoea. $\bigcirc=$ diabetics and $\square=$ patients in whom a firm diagnosis was made on clinical grounds but who did not fulfil the criteria for 'proven' pancreatic disease.

rhoea associated with villous atrophy had normal levels (fig 5), including 12 with adult coeliac disease.

\section{Pain}

Most patients with chronic pancreatitis or carcinoma pancreas $(12 / 20)$ but only a few of those with acute or relapsing disease $(3 / 13)$ had abnormal mean tryptic activities. The only control patient with an abnormal mean tryptic activity and pain had a lymphoma of the terminal ileum but no other findings to suggest pancreatic dysfunction.

\section{Other symptoms}

The distribution of results in this group was unusual. The mean tryptic activity was normal in all six patients with 'proven' pancreatic disease whereas it was abnormal in five of six patients with 'suspected' pancreatic disorders.
REPEATED LUNDH TESTS

There was close agreement between repeated tests in patients without pancreatic disease (14 patients, 17 repeat tests, $\mathrm{r}=0.82, \mathrm{P}<0.001$, test interval from a few days to several years). Clinical deterioration of pancreatic function in four patients with 'proven' pancreatic disease was reflected by falling levels in serial tests.

\section{FALSE-POSITIVE RESULTS}

There were only six false-positive results in 523 tests and four were caused by faulty positioning of the sampling tube. Repeat tests in these four patients with the sampling tube correctly placed gave normal values. Another patient had a gastrinoma of the duodenum with excessive gastric secretion (table I). The remaining patient has been discussed previously (see section headed pain).

\section{Discussion}

Most tests used in the diagnosis of pancreatic disease are time consuming and the results may be difficult to interpret. The Lundh test has been reported to be a simple, yet accurate method for diagnosing exocrine pancreatic insufficiency in selected patients (Lundh, 1962; Cook et al, 1967; Levin et al, 1972; Lurie et al, 1973). We have confirmed and extended the earlier findings by analysing the results of tests performed on a much larger unselected population of patients, who were referred by several clinicians whenever pancreatic disease was suspected on clinical grounds. The number of tests reflects the high index of suspicion for pancreatic disease among the doctors and the ready availability of the procedure, while the low incidence of technical failures underlines the simplicity of the technique. We also found that additional data of diagnostic value may be obtained from inspection of the duodenal fluid.

Previous work from this department has suggested (from data on 14 normal subjects and 30 patients without pancreatic disease) that the lower limit of normal for mean tryptic activity was 10 IU/1 (Cook et al, 1967). The present results, however, based on 235 patients without evidence of pancreatic disease or diabetes and who had not undergone gastric surgery, have shown that acceptance of this level would result in an unacceptable number of falsepositive results. On the other hand, if the tube was correctly placed in a patient without previous gastric surgery a mean tryptic activity of $6 \mathrm{IU} / 1$ or less was diagnostic of pancreatic exocrine deficiency, only one of the 235 control patients having an abnormal value. By contrast, $71 \%$ of patients with chronic pancreatitis or carcinoma of the pancreas had a mean tryptic activity of 6 or less. This result 
is particularly encouraging because the criteria used for defining 'chronic' pancreatitis in the present study merely imply some structural damage, not necessarily associated with exocrine insufficiency, whilst carcinoma of the pancreas is not always accompanied by a disturbance of pancreatic exocrine function. The present study also confirms earlier findings that steatorrhoea associated with a mean tryptic activity of $2 \mathrm{IU} / 1$ or less is invariably due to pancreatic exocrine insufficiency whilst patients with an abnormal jejunal mucosa (including coeliac disease) usually have mean tryptic activities above $6 \mathrm{IU} / 1$.

The Lundh test was of practical value in the management of patients presenting with jaundice, as it was often possible to deduce the site and the benign or malignant nature of the obstruction from the appearance of the duodenal contents and the mean tryptic activity. Absence of bile from the duodenal aspirate throughout the two-hour period almost always indicated a malignant lesion, but whilst patients with carcinoma of the hepatic or bile ducts had a mean tryptic activity $6 \mathrm{IU} / 1$, most patients with carcinoma of the head of the pancreas had an abnormal mean tryptic activity. The latter diagnosis was sometimes suggested by the failure of the tube to progress beyond the second part of the duodenum associated with absence of bile in the samples. In marked contrast bile was rarely absent from the duodenal contents in patients with obstructive jaundice due to benign causes. On the other hand the Lundh test alone cannot differentiate chronic pancreatitis from carcinoma of the pancreas but a review of all clinical and laboratory findings usually gives the answer. This difficulty may also be experienced with the secretin/pancreozymin test (Braganza and Howat, 1972) and even laparotomy may not give the answer in some cases (Trapnell, 1971).

When pain was the sole presenting symptom the mean tryptic activity did not quite so clearly differentiate pancreatic from other gastrointestinal diseases. This was not surprising since pancreatic damage was less extensive in this group and the patients had no clinical evidence of pancreatic dysfunction. Nevertheless the Lundh test was diagnostic in $60 \%$ of patients with chronic pancreatitis and carcinoma pancreas.

In patients who present with pain or jaundice there remains a region between 7 and $10 \mathrm{IU} / 1$ where the mean tryptic activities of patients with and without pancreatic disorders overlaps. This is disappointing but not unexpected. The range of 'normal' pancreatic function in patients is wide. Protein deficiency can influence pancreatic exocrine function (Véghelyi and Kemény, 1962; Tandon, George, Sama, Ramachandran, and Gandhi, 1969; Tandon,
Banks, George, Sama, Ramachandran, and Gandhi, 1970), and disturbed protein metabolism is likely to occur in some gastrointestinal diseases. Wormsley (1972b), for example, attributes the low preoperative pancreatic exocrine function in patients with gastric ulcer to possible malnutrition. Moreover in the absence of duct obstruction considerable pancreatic damage may be present before abnormal exocrine function becomes apparent. Other tests of pancreatic disease which rely on pancreatic exocrine function do not appear to be more helpful in this situation. We have found that the Lundh and secretin/pancreozymin tests give comparable results (Waller, Kapp, Mottaleb, Noguera, Kellock, and Wiggins, 1972; Kapp et al, in preparation) and other workers report similar findings (Lurie et al, 1973). Duodenal levels of radioselenium following intravenous $\mathrm{Se}^{75}$ methionine also correlate well with the mean trypsin concentration but do not yield additional information (Youngs, Agnew, Levin, and Bouchier, 1971a), whilst pancreatic scans rarely increase diagnostic accuracy unless a localized lesion is present and false-positive scans are not infrequent (Youngs, Agnew, Levin, and Bouchier, 1971b; Bouchier, 1972).

We have found the Lundh test to be a practical procedure involving only a simple intubation with a single-lumen tube, which is well tolerated by sick patients. Vomiting is unusual but some workers prefer to give metoclopramide before intubation (Levin et al, 1972). Although this drug apparently has no effect on pancreatic secretion in animals (Delcourt and Wettendorff, 1968), there are no data in man at present and therefore it is probably advisable either to give metaclopramide to all patients having a Lundh test or to none. As no injections are required, medical supervision can be limited to screening the tube at the beginning and end of the study, or during the test if the nurse suspects the tube may have slipped back. The cost of the materials is low (about $0.7 \mathrm{p}$ for each Lundh meal) and no elaborate equipment is needed. Specimens can be stored at $-20^{\circ} \mathrm{C}$ without deterioration for at least four weeks (Cook et al, 1967) and require only one duplicate estimation. The diagnostic success rate is very reasonable and false-positives are unlikely if the sampling tube is correctly positioned. We would suggest that the Lundh test is suitable for the diagnosis of pancreatic insufficiency in gastrointestinal units as well as in general hospitals. It should be borne in mind that it is not suitable for basic physiological studies of pancreatic function.

We thank our medical colleagues from the Central Middlesex, Middlesex, West Middlesex, Royal Free, and St Mary's (Paddington) Hospitals for referring 
their patients for the test. Our thanks are due to Staff Nurses D. O'Neill, D. Elcock, and S. Pittkin for supervising the tests; to Mrs Wendy Lamble and Mr Raymond Sapsford for technical assistance, and to Mrs P. A. Stuart for typing the manuscript.

\section{References}

Bouchier, I. A. D. (1972). Difficulties in the laboratory diagnosis of pancreatic disease. Proc. roy. Soc. med., 65, 845-846.

Braganza, Joan M., and Howat, H. T. (1972). Cancer of the pancreas. Clin. Gastroent., 1, 219-237.

Chey, W. Y., Shay, H., and Shuman, C. R. (1963). External pancreatic secretion in diabetes mellitus. Ann. intern. Med., 59, 812-821.

Cook, H. B., Lennard-Jones, J. E., Sherif, S. M., and Wiggins, H. S. (1967). Measurement of tryptic activity in intestinal juice as a diagnostic test of pancreatic disease. Gut, 8, 408-414.

Delcourt, A., and Wettendorff, P. (1968). Metoclopramide and exocrine pancreatic secretion. (Abstr.) Gut, 9, 257.

Levin, G. E., Youngs, G. R., and Bouchier, I. A. D. (1972). Evaluation of the Lundh test in the diagnosis of pancreatic disease. J. clin. Path., 25, 129-132.

Lundh, G. (1962). Pancreatic exocrine function in neoplastic and inflammatory disease; a simple and reliable new test. Gastroenterology, 42, 275-280.
Lurie, B., Brom, B., Bank, S., Novis, B., and Marks, I. N. (1973)。 Comparative response of exocrine pancreatic secretion following a test meal and secretin-pancreozymin stimulation. Scand. J. Gastroent., 8, 27-32.

Tandon, B. N., Banks, P. A., George, P. K., Sama, S. K., Ramachandran, K., and Gandhi, P. C. (1970). Recovery of exocrine pancreative function in adult protein-calorie malnutrition. Gastroenterolog., 58, 358-362.

Tandon, B, N., George, P. K., Sama, S. K., Ramachandran, K., and Gandhi. P. C. (1969). Exocrine pancreatic function in proteincalorie malnutrition disease of adults. Amer. J. clin. Nutr., 22, $1476-1482$.

Trapnell, J. (1971). The operative diagnosis of carcinoma of the pancreas. Brit. J. Surg., 58, 849-851.

Véghelyi, P. V., and Kemény, T. T. (1962). Protein metabolism and pancreatic function. In Ciba Foundation Symposium on the Exocrine Pancreas., edited by A. V. S. de Reuck and M. P. Cameron, pp. 329-352. Churchill, London.

Waller, Sheila, L., Kapp, F., Mottaleb, A., Noguera, E. C. A., Kellock, T. D., and Wiggins, H. S. (1972). A preliminary comparison of the Lundh and secretin-pancreozymin tests in the diagnosis of pancreatic disease. (Abstr.) Arch. Mal. Appar. dig., 61, 227C.

Wiggins, H. S. (1967). Simple method for estimating trypsin. Gut, 8, 415-416.

Wormsley, K. G. (1972a). The effect of vagotomy on the human pancreatic response to direct and indirect stimulation. Scand. J. Gastroent., 7, 85-91.

Wormsley, K. G. (1972b). Pancreatic exocrine function in patients with gastric ulceration before and after gastrectomy. Lancet, 2 , 682-684. 